soften too soon; I have known it do so. I have known it happen that a ferv hours after an operation the catout sutures have disappeared and the abdominal viscera have come out through the wound. But what has happened more often is that they have come out underneath the skin, having escaped from the abdominal cavity itself. And that often leads to disaster, always necessitates another operation to close the round again, and very probably the administration of an anaesthetic a second time and under unfavourable conditions.. As a matter of fact the procedure has boen attended with many accidents - that is to say, with loss of life. I also advise you not to use a continuous sinturc. Use interrupted sutures for each of the five layers. It takes longer, I admit, but the advantage is that each snture is self-contained; it does not depend for its efficiency upon what happens to any other part of the thread, whereas in the other case the whole line of suture is dependent upon every individual portion of it. Use interrupted sutures, and then, if by any chance one should havpen to get untied or softened or if one should get infected, it is merely a localized failure, a localized injury which has been done, and not one which affects the whole suture. When a continuous suture goes wrong failure of one spot affects for evil the whole of that suture.

In conclusion, a word about the after-treatment of these caises. The one thing I want you to remember about the surgery of the abdominal wall is that this structure is essentially a great and important muscle. Now an injured munscle should not be subjected to early strain. The tendency, in the rush and hurry of surgical hospital practice, is to get patients up too soon, to lumry them out ind to think that when the skin edges are tolerably firm the whole wound is repaired. That is a grave mistake. $A$ very important muscle is involved in every incision through the abdominal wall, and the full and complete repair of it is an extremely important incident in the patient's life, and you must guard this injured muscle against undue strain by keeping the patient in bed a sufficient time, that time being dependent upon the length and character of the incision, the size of the belly, and the general nutritive activity of the patient. You must also take into con. sideration any special circumstances of the case, such as the strain involved in coughing and in the patient's occupation. Let me repeat, then, that the abdominal wall is one of the most important muscles in the whole body. All surgical interference with it should be thoughtfully and skilfully planned to preserve the integrity of this muscle and its nerves. The elosure of the wound should be so conducted as to restore it to its original form with the utmost accuracy, and the after-treatment should aim at protecting the weakened muscle from all undue strain.

THe report of the Surgeon-General of the United Sitates Army issued on September 1st, 1911, shows that the mean strength of officers for 1910 was 4,216 . There were 2,515 admissions, 15 cleaths, and 119.54 constantly non-effective from all causes, equal to ratios 596.54, 3.40 and 28.35 , as compared with ratios of $628.90,4.20$, and 30.27 per 1,000 for 1909. The admission and constantly inon-effective, and death rates were lower than for 1909 . The principal causes of admission in the order of their respective importance were: Influenza, 51; diarrhoea and cnteritis, 40.09; bronchitis, 37 ; tonsillitis, 28.46; dyspepsia, 26.33; coryza, 24.67; and malarial fever's, 19.21 per 1,000. Thirty-seven officcrs were retired on account of disability during the year-a rate of 8.38 per 1,000 , compared with 11.91 for 1909 . The mean strength of enlisted men, white and coloured, for 1910, was 71,534. The total number of "admissions to sick report" was 67,940 , equal to an admission-rate of 949.76, as conıpared with 1,062.99 for the previous year. The deaths from all :auses numbered 320 , of which 175 were from disease. The death-rate from all canses was 4.46 , and 2.44 for iliserse, as compared with 4.91 and 3.02 respectively for the preceding year. The death-rate from disease is said to be the lowest on record in the office of the Surgeon(iencral. The discharges for disability numbered 1,007 , the rate being 14.02 , as compared with 16.84 for 1909. The artmission-rate for disease in 1910 was 766.06 per 1.000 of mean strength, for the previous year 865.92, and for the decade 1,248.77. 'A chart shows that the health of the Inited States Army has constautly improved since 1899, when the admission-rate for disease alone was 2,125.74.

\section{An Adtreas}

or

\section{CER'TAIN OPERATIVE PROCEDURES IN THE PARALYSIS OF CIILDREN, \\ WITH SPECLA REFERENCE TO POLIOMYEITLS.}

\author{
DeLIVERED BEFORE THE \\ Northumberland and Durhay Medical Socifity,
}

BY

ROBERT JONES, Ch.M.LPoo, F.R.C.S.EdiN.,

LECTURER ON OITHOPAEDIC SURGENY, LIVERPOOL VNIVERSITY.

- Mr. President and Gentlumen,-Before dealing with the operative side in the treatment of poliomyelitis it is necessary that we should understand certain fundamental principles the neglect of which renders either operative or: mechanical work ineffective, and ignorance of which leaves us quite untit to judge as to whether an operation be necessary or not.

Some Pathological Considerations.

In the first place we must learn to distinguish between a paralysis due to destruction of a motor cell in the anterior cornu, and that due to the mechanical disability of a muscle which remains inactive after the recovery of its governing cell.

We have been tanght that a muscle which exhibits the reaction of degeneration is in such a state that it cannot recover voluntary power of response. Many years ago I drew attention to several recoveries in muscles which displayed this reaction, and this has been borne out by many observers, including Hoffa, Konig, Lange, and others, and has been proved both clinically and histologically by Koch.

We may look upon it as a certain and valuable fact that if a muscle reacts to faradism it will recover, but the contrary proposition does not follow. Sherren has certainly shown that, very carefully taken, galvanic reaction will show whether a muscle has been completely severed from its motor nerve cell or not, but in practice it is next to impossible to -make this observation quite thorough.

Acute poliomyelitis of course often permanently destroys motor cells in the anterior horns of grey matter, and the muscles dependent upon them are for ever useless unless nerve transplartation in the future may come to their rescue. This complete destruction, however, is fortunately the rarer condition, and from the clinical evidence we have of rapid and complete recovcries from complete paralysis, and the very many partial recoveries, it would appear that the cells have generally suffered from temporary injury rather than from destruction.

With the irrecoverable muscle the surgeon has no direct interest; his attention is directed to the cells not actually destroyed, althongh the muscles governed by these cells may apparently be partially or hopelessly paralysed. It would be highly speculative to discuss the reasons why the recovered cells fail to secure control of their dependent muscles. Something lias happened to make the muscle incapable of responding to a motor stimulus. Whether no afferent muscle sense stimulus is sent back to the nerve cell in consequence of this, or whatever be the reason, it is clinically certain that the nerve cells become inactive, though not extinct, and with appropriate treatment of the muscle or group of muscles, functional activity can be reinstated.

Overstretching of Muscles.

When a muscle governed by a live cell fails to act, we may call it a case of functional disability. Now, what is the cause of functional disability. in a muscle? The great outstanding cause is overstretching. The surgeon has to find out the difference between a truly paralysed musclo and a muscle overstretched, and herein lies the fundamental principle which should guide us in the treatment of infantile paralysis. If the practitioner from the first disappearance of acute: symptoms could prevent the weak muscular groups from being stretched, paralytic disabilities and deformities would be comparatively rare. As it is, too little attention is paid to the posture of the child during 
his convalescence from the aciute onset. For instance, the child lies with the wrist flexed, and the flexor muscles con. sequently undergo some adaptive shortening, while the extensol's are overstretched and placed at a mechanical disadvantage. As the poliomyelitis in the corresponding part of the cervical enlargement begins to pass off, the flexor tendons, from their position of advantage, are in a condition to respond to the first stimuli which come from their recovering motor cells, while the extensors, placed at disadrantage, cannot. Dropped wrist is a convenient type of paralysis to illustrate this doctrine of the functionally disabled muscle, of which $H$. $O$. Thomas wrote a quarter of a century ago.

I have on other occasions afforded instances, expcri. mental and clinical, in proof thiat drunkard's palsy is not always due to direct pressure upon the musculo-spiral nerre, and that it frequently occurs when a patient lies with his hand twisted under him in acnte flexion, is due, in fact, to continued tension of muscle fibre. Numerous cases of different types could be adduced in proof of the paralysing influence of muscle tension. For instance, a man cut his wrist, dividing all his flexor tendons. His doctor stitched up all the tendons, and, in order to prevent strain, very naturally fixed the wrist in extreme flexion. Some weeks later lie was sent to me because he had no power in the extensor muscles. Take, again, as an instance, dropped wrist from lead poisoning. Thic lesion may be either in the cord or in the nerve trunlis ; in either case the muscles cease to be controlled by their nerre centres, and the patient goes about with his wrist flexed by gravity. while the extensor muscles become disabled by continuous overstretching. This condition is usually bilateral, and if the case be of some weeks' standing, an interesting experiment may be made by placing on one of the paralysed arms a splint to keep the hand dorsiflexed. Recovery will be. much more rapid on the side on which the extensors are thus relieved from strain than on the other side. The treatment in all such cases is to keep the wrist and fingers in extreme dorsi-flexion. Felieved of strain they undergo adaptive shortening and soon begin to respond to the constant stream of tonic stimuli sent to them by afferent and subcortical centres, and they then come under the higher control of the voluntary centres.

We will further consider some of the factors which assist in determining the persistence of functional disability in certain groups of muscles. Take, for example, the paralysis of the muscles supplied by the external popliteal nerve. This is a common type of residual paralysis producing paralytic talipes equino-varus. This state is often determined by pressure of the bedclothes, that is. it is a deformity due to decubitus. Again, if a patient be left lying.with the knees and hips flexed, and thighs rotated inwards, the flexors contract, and the extensors remain weali or functionally disabled. And I could go on indefinitely giving illustrations to prove that most of the recognized types of residual paralysis may arise from incorrect posture in bed, easily preventable by a little thought and trouble. If this were given, muscles might be saved from becoming atonic. The normal efferent motor and afferent tonic impulses between the muscles and the nerve centres would be re-established sooner, with probably a favourable reaction on the recovery of the nerve cells.

Another cause of persistence of functional disability may be found in unegual degree of recovery in opposing mus. cular groups. The stronger group is apt to overpower the weaker, which, giving way in the mequal struggle; becomes functionally powerless. Or the same result may arise from the fact that certain nerve cells governing one group of muscles may recover earlier than those governing a second group, and thus a start is given to the one at the cxpense of the other.

Perhaps the most potent factor for evil is what I have called the erroneous deflection of body weight. It operates chiefly in the lower limbs. One instance will illustrate it. If there be weakness in the tibial group and the patient be allowed to walk, the valgoid deformity will constantly increase, the tibial muscles be more and more stretched, adaptative contraction will affect the peronei and structural alteration in the tarsal bones, all of which might be avoided by perhaps a little alteration in the heel of the boot to deviate pressure from the inner to the outer side of the tarsus. I wish you carefully to bear in mind this question of strain upon a weak tendon, as it so closely affects us when we deal with tendon transplantation.

In the milder type of case where we are called to dcal with a group of museles where the power is but slightly impaired, the muscular balance may be restored by orerdeveloping the weaker group. This can only be done by massage and exercises if there are no fixed deformitics. For instance, suppose the peronei are weak, but the foot can quite easily be placed everted, then massage and exercises may be expected to: succeed. But if the foot cannot be everted because of adaptive shortening of the tendons, then massage and exercise of the peronei are useless until the deformity is first corrected. This is a principle I would apply to other departments of orthopaedics, such as scoliosis, flat-foot, and congenital talipes, where inequality in muscular power is present-from whatever cause. From a mild case we may go on to cases of every degree of severity-from such mild cases as I have just discussed onwards to cases of flail ankles, of complete paralysis, and finally to cases with bony deformities requiring osteotomies, exsection of bony wedges, arthrodesis, or tendon transplantations.

Late Recovery of Muscle Pouer.

But before any operations are performed affecting the mobility of a joint every use should be made of available muscle power. No surgeon should operate on these cases until he has fully satisfied himself whether it be possible or not to restore the apparently paralysed musclc. Neglect of this precaution produces the distressing phenomenon of the unexpected recovery of muscles which wero ignored as paralysed. For example, a few weeks ago a patient was brought to me with a partially ankylosch knee. The operation of arthrodesis had been performed, with a view of bringing about a bony ankylosis. As an arthrodesis the operation had failed, for the knee had a short range of movement. As an experiment illustrating the principle we are discussing it was very successful, because the quadriceps was acting with considerable strength. Prolonged fixation had relieved the quadriceps from all strain, and restoration of function resulted. This case exemplifies the mistake of taking for granted that a muscle is paralysed without first finding out if it be really paralysed.

The first stage of treatment is the correction of existing deformity, and when deformity is corrected the limb should be kept immorable until the ligaments, muscles, and even bone have become of normal length and shape. To break the continuity of treatment is to bring about a relapse. ' For instance, suppose a drop wrist is placed in a splint designed to dorsiflex the hand and wrist. The hand must not bo allowed to flex until recovery has occurred. While the patient washes the hand must be held dorsiflexed. This point is fundamental, and neglect to observe it means failure. The slightest stretching of a muscle on the point of recovery disables it agaiu, and all the good work of weeks and months may be thwarted by a single act. I cannot cmphasize this point too strongly.

The clinical test of the recoverability of a muscle therefore depends on an experiment. Let it be kept for a prolonged period, perhaps six, sometimes twelve montlis, in a position of relaxation. This test should be made before condenming any muscle, no matter for how many months or years it may apparently have been paralysed. When. therefore, one sees in textbooks that we are to despair of the return of power after this length of time, we can quite well afford to ignore the advice unless added to the time test therc has been uninterrupted musenlar relaxation.

Dr. Wittek (of Graz), in an article of which he sent me a reprint some time ago, offers very convincing evidence of the truth of the correctness of the principles $\mathbf{I}$ advocate. His paper dealt with the great epidemic of poliomyelitis in Styria in 1907, and he relates the case of a boy who during it suffered from poliomyelitis. Twelve months later his parents brought him to the neurological department, fron which he was transferred to the surgical department, with a note that all the muscles of the left leg supplicd by the external popliteal nerve were paralysed, did not respond to faradic stimulation, and showed the reaction of degeneration. As this condition was found a full year after the onset of the disease, as the foot was in the equino-varus: position, and only the muscles of the calf werc acting, it 
was decided to perform tendon transplantation, after correcting the deformity. The position of the foot was first overcorrected, and it was put up in plaster-of-Paris for a fortnight. Then the tendo Achillis was lengthened, and the flexor longus hallucis was converted into a pronator, and the flexor longus digitorum into a supinator by Lange's method. The patient was then kept in bed for six weeks in plaster-of-Paris, and afterwards allowed to walk in a plaster-of-Paris splint for another six weeks. He was then provided with boots with lateral support, and an elastic band to dorsiflex the foot. He was next seen in July, 1909, a year after the operation, when he was brought because the foot was in extreme valgus position, with the internal malleolus touching the floor. On testing for function, the astonishing result appeared that now all the muscles supplied by the external popliteal nerve could be actively contracted, and had recovered full function. The muscles reacted promptly to faradic stimuli, and the galvanic reaction was normal. The deformity could only be attributed to the power added to the pronators and subtracted from the supinators by the operation. If nemoplasty had been performed in July, 1908, the surgeon would have had a brilliant result to show. There was nothing for it but to attack the existing deformity. The position was first corrected, and then the tibialis anticus as well as the flexor longus digitorum, which had been converted into a pronator, were both shortened. As before, the foot was put in plaster in a supinated position for twelve weeks. After this time the plaster was removed. The foot remained in supination; no voluntary movement of pronation could be performed. Faradic stimulation prcduced no contraction in the peronei muscles.

The conclusion (says Dr. Wittek) to be drawn from this observation was inevitable. The paralysed muscles were functionally disabled by overstretching. 'The second part of our case has the great advantage of being an unintentional experiment.

I have not only to thank Dr. Wittek for giving a striking illustration of principles which I have been advancing for years, but also for the generous reference he makes to $\mathrm{my}$ work. He announces his intention of waiting in future for recovery of muscles under appropriate treatment before proceeding to tendon transplantation.

Before performing transplantation of tendons or arthrodesis preliminary osteotomies are sometimes required. For example, knock-knee is frequently associated with paralytic conditions of the lower limb, and it is necessary to correct this by osteotomy to adjust the line of traction of the muscles before transplanting some of the ham. strings into the patella. Again, as a knock-knee means erroneous deflection of body weight, it should be corrected before proceeding to arthrodesis of the ankle in a case where the ankle is a hupelessly flail-like joint. In both instances the object is to adjust the alignment of the limb and to get the transmission of body weight true before proceeding to the more delicate operation.

The age of the patient should guide us in our mode of attack upon these preliminary deformities. In very small children, in whom the structures are soft and yielding, gradual correction of deformity is to be preferred to the more rapid methods which may be employed in older children. In young children there is no object in hasty transplantation of tendons. We should correct the deformity, provide the simplest instrument to keep the limb in good position, prevent erroneous deflection of body weight during walking, and let the child grow and develop until it is about 5 before transplanting its tendons, and 10 before doing an arthrodesis.

\section{Treatuent}

I will now briefly indicate the order in which we should attack paralytic deformities.

I. Reposition, Manipulation, Supports.

I would first endeavour to correct usu cterormity by reposition, manipulation, and mechanical supports. For instance, in cases of valgus deformity at the ankle, the inner side of the ankle should be made higher than the outer, an iron should be placed between knee and ankle on the outside, ending in the heel of the boot, and should not have a joint opposite ankle. This supports and inverts the ankle. allowing overstretched ligaments to shorten and overstretched muscles to recover power if they can.
Similarly, in cases of talipes equinus, the iron is let into the back of the heel and extends forwards into the wais of the boot to keep the foot at right angles and relax the weak extensor group.

\section{Division of Contractions.}

Eands of fascia and contracted tendons which prevent correction of deformity may have to be divided. In paralytic club-foot, the Achilles tendon, the plantar fascia, the peronei, or any obstructive band may require subcutaneous division. In the thigh the tensor fascia may require division. This is best done by open incision. In the knee böth the popliteal fascia and the hamstrings may require open division, but if the hamstrings are necded for transplantation later an effort should be made to spare them from division.

\section{Removal of Skin Areas.}

Parents are often careless in carrying out instructions loyally and in detail. They do not see the importance of keeping a foot constantly and for a prolonged period in one position when we require uninterrupted relaxation of muscle. They allow the child to remove boot and iron to have a run barefooted. Under such conditions results are variable and disappointing. To put $\dot{a}$ stop to this in paralytic equinus I began about ten years ago to remove an area of redundant skin sufficient to keep the foot at right angles when the wound was sewn. In equino-varus the skin is removed over the outside and front of the ankle. In equino-valgus from the front and inside. In ten days the stitches may be removed, and the foot will retain a correct position for some months, even if parents do carelessly remove splints. So satisfactory has this operation been that it forms part of my routine treatment of small children, for protecting weak or functionless muscles from strain. Though I planned this particular operation for the prevention of muscular strain, I had several years previously started to remove large shin areas in those cases of paralysis of the upper arm where the elbow and shoulder were paralysed and the hand acting. By fixing the forearm to the upper arm, the hand could be utilized for feeding purposes. I have already published several instances in which recovery has taken place in the biceps and brachialis anticus some months after this operation has been performed-a further testimony of the pleasing results of muscle relaxation.

\section{Tendon Transplantation.}

We will now turn our attention to tendon transplantation, and we must first answer the question as to when we are justified in transplanting a tendon. My answer is, not before twelve months have been spent in endeavouring to promote the regeneration of the paralysed muscle, and only rarely in children under 5 years of age. It may be performed for many different purposes.

(a) To reinforce a weak muscle-for example, a ham. string may be transplanted into the patella to reinforce a weak quadriceps which ineffectually struggles against strong flexors, and is in danger of relapsing into inactivity. Or, again, where weak inverters of the foot may be reinforced by a transplanted extensor proprius hallucis. These are both instances where a partially acting muscle derives help from a healthy one.

(b) Again, transplantation may be performed to replace a muscle which is quite inactive and does not show any signs of recovery under conservative treatment. For instance, the peronens longus may be implanted into the scaphoid or first metatarsal, to act as an inverter of the foot. In this way an extreme valgus deformity mav be converted into a well-balanced foot requiring no support.

(c) Another interesting type of transplantation is illustrated where the foot becomes inverted especially at the heel. To overcome this I split the tendo Achillis in two, separate the inner half from the os calcis, pass it under the outer half and insert it to the outer side of the heel. This amounts to a change of insertion and involves no psychological problem. Experience of the operation of transplantation of tendons has shown me that success depends upon the recognition of the principles we have already discussed and in attention to many. details. The details are generally details of technique and deserve some consideration. The transplantation, wherever possible, should be tendon to bone. A tendon inserted into soft parts seldom holds firmly; the periosteum is often so thin and 
difficnlt to separate that a satisfactory union is ont of the question. Where it can be brought about the bone should be tunnelled and the tendon passed through the tunnel and securely fixed. This can be usually satisfactorily performed when the ball of the too requires lifting, as in pes cavus, where the proprius hallucis is passed through the metatarsal head, or in the fixation of the peroneus longus to the inner-side of the tarsus.

If the tendon is too short to pierce the bone, as in the case of the tibialis anticus, a piece of silk is tied rery tightly round the tendon, about half an inch from its cut end, so tight that the tendon will neither fray nor slip; a cleft or groove is made into the bone, and a strong periosteum needle. threaded with chromicized gut is passed through the soft bone and throngh the tendon just above the ring of silk, so placed that when the chromic suture is tied the tendon will be embedded in bone. To make it still more secure a short nail is hammered through the tendon into the bone.

When bringing a tendon from one part of the limb to another, it should pass in as straight a line as possible, aroiding any bend or angle, which might obstruct its action. Where possible the sheath should be taken with the tendon, but frequently this cannot be done. Fat is the best medlium for the transplanted tendon to trarerse.

My most generally successful transplantations are in this order :

Tibialis anticus to the outer tarsus in paralysis of the peronei.

Peroneus longus into scaphoid for paralysis of the tibial group.

The extensor proprius hallucis into any part of the foot where it may be wanted.

Hamstrings to patella, to reinforce the quadriceps, provided the strictest after-treatment can be secured.

Deflection of part of the tendo Achillis to onc or other side of the foot.

In transplanting the hamstrings into the patella I nsually confine myself either to the use of the biceps or to the semitendinosus and semimembranosus. When I use the inner hamstrings I stitch the semimembranosus to the semitendinosus in order to have a good loug tendon and lissect the muscles well up the thigh in order not unduly to deffect them. An oblique incision about 2 in. long is made from the upper part of the patella in the line the transplanted muscle is to travel. A blunt dissector is in thiir case passed beneath the fascia into the popliteal incision and a wide tunnel secured for the passage of the tendon. At this stage care must be taken lest the trans planted tendon is twisted on itself during transit. A small opening is then made just above the patella, and another, three-quarters of an inch behind. A forceps is introduced from below upwards through both openings, and the tendon is led through them to be fixed to the upper surface of the patella. The sartorius has too fleshy an insertion to be of innch use to ns.

Again let me reiterate the necessity of first correrting all deformity before transferring the tendon; to give the tendon plenty of lateral play during its transit and to prevent it from being unduly strained during the stage of recovery.

\section{Arthrodesis.}

Arthrodesis may now be considered as one of our operative procedures. It consists in removing the cartilage covering the joint surfaces of bones and producing a firm ankylosis. The joints which best lend themselves to this operation are the ankle and mid-tarsal joints. At the ankle and mid-tarsal the object of the operation is to obtain a secure and firm base of stopport in walking. The operation of arthrodesis, therefore, should not be considered until it is ascertained that muscular power is not available to produce a well-balanced ankle by any other means. Thie surgeon, therefore, is never in a hurry to perform arthrodesis, for it is his duty first to correct deformity; to take measures already indicated to conserve and develop any power remaining in the muscles controlling the foot and ankle. After this he must decide whether the patient will be better off with a stiff foot or with a weak and movable ankle supported by apparatus. This is often a matter of social station. In the rich, an inefficient and mobile ankle may be made useful by mechanical aid, while in the poor a firm, immobile joint is of more use and of less expense. Much also will depend upon the surgeon.
Recent developments in general surgery have so simplified and perfected the art that once an operation is completed the trouble ends. In orthopaedics, however, the surgeon? task: often only begins when the operation ends, and, despite the many fascinations of this special work, very patient industry and ingenuity are always in demand.

At what age should arthrodesis be done? I cannot remember a single case of good bony union in a case operated upon under the age of 4 . Of children operated upon under 7, not one-fourth have satisfactory stability in ankle or foot. In cases over 10 years of age the tixations are almost universally good.

From every point of view, early opcration is a mistake in these paralytic cases. Interference with very young bone results in weak fibrous union. and impaired or irregular growth, with consequent new deformities, which require treatment at a later stage. Under no circumstances should an arthrodesis be performed until the child has passed his eighth year. There is plenty to be done during the waiting, and if this be done wcll, perliaps no operation will be needed. Before he is 8 , his existing deformities will have been corrected, skin areas will have been removed, to relieve functionless muscles of strain his body weight will have been appropriately deflected, and he will have been to walk in splints, swinging his limb about, and using it effectively in a correct position, so that exercise becomes a powerful agent in encouraging growth, physiological and functional development.

To illustrate the mode of operative procedure, let us assume that we are dealing with a case of paralytic equinus, in which the paralysis is so extensive that there is no possibility of dealing with the case by any form of tendon transplantation, and that a stiff ankle has been definitely selected. By an incision over the front and outer side of the ankle the joint is reached. The incision should extend from just above the ankle-joint to a Jittle beyond the head of the astragalus. The ankle-joint is opened and well exposed, and with a chisel the cartilawe is removed from the astragalus, the tibia and the inner aspects of the malleoli. At this stage we must malie allowance for any associated valgus or varus deformity, at the ankle. To provide for the correction of such lateral deformity, a wedge-shaped slice of bone is taken from the top of the astragalus with the base to the inner or outer side according as we wish to invert or evert the foot. When the cartilage has been remored from the sides of the astragalns, and the inner aspects of the malleoli, it is sometimes found that the astragalus will not confortably fit the gap between the malleoli. It is well in these cases to introdnce a fine chisel through the womd and divide each malleolus at its base and squeeze them both against the astragalus.

The mid-taisal joint between the astragalus and scaplioid is attacked in the same way at the same operation. The foot is then brought up to a right angle, the cut surfaces of bone being well pressed together. The foot is then fixed in correct position in a club-foot shoc and ten days later in plaster-of-Paris.

In operations upon the tarsus it is well to know beforehand the growth deformity which sometimes occurs. It is nearly always a pure varus, and it is therefore advisable to aim at leaving the foot very slightly in a valgus position. When the union is complete, in six or seven weeks' time, the child is allowed to walk, but the union must be carefully guarded from strain. He must be seen at intervals for fear incorrect deviation of body weight be spoiling the result.

In another type of case it is better to leave the midtarsal joint free and arthrolese the ankle only. This is indicated when there is but the slightest power in the extensors of the toes, which would never recover sufficiently to oppose the weight of the dropped foot. Such weak muscles might, however, be quite able to retain their function when required to act only through the more limited range of movement at the mid-tarsal joint.

The ankle and mid-tarsal joints are those best suited for arthrodesis, and next to these the shoulder. The object of arthrodesis of the shoulder is to make the humerus move with the scapula as one bone. It is, therefore, only of service when there is good muscular control of tle scapula. It is only indicated when all the muscles controlling the shoulder-joint are paralysed. If the missing 
of the hand are inactive, there is no object in an arthrodesis of the shoulder.

I perform the operation by the anterior incision commonly, used for excision of the shoulder-joint. The hinierus should be fixed in abduction and internal rotation -so that the hand can be brought to the mouth.

It is very rare for me to perform arthrodesis of the knee. It is an operation which, if not performed early, gives an admirably firm limb, and I have patients who go about their work contentedly, with a stiff knee and ankle; others, however, regret the day on which they consented to this operation. This regret is merely due to inconvenience. The limb, they say, is always in the way and in evidence. Where money permits, a lock calliper fills every indication, and can be used for walking in extension or sitting in Hexion.

Arthrodesis, in my opinion, is quite unsuitable for hip, wrist, or elbow. At the elbow because the removal of skin and fixation of forearm at an acute angle does what is desired. At the wrist the usefulness of the fingers depends on the presence of some degree of co-ordination of inovement, which means that both extensors and flexors must be acting and controlling each other. If there be power in the muscles the hand can always be made useful hy means other than arthrodesis, and, of course, if the hand is useless the wrist should be left alone.

\section{Trinsplantation of Nerves.}

No discussion of the surgery of paralysis would be complete at the present day without some reference to the traisplantation of nerves. Our present knowledge of regeneration of nerves is such that it-seems entirely reasonable to expect that if the distal end of a paralysed motor nerve be connected by grafting with the proximal end of an active motor nerve, it should result in restoration of conductivity in the paralysed nerve, and of function in the muscles supplied by them. This operation is not new, for Hackenbruch reported a successful case of grafting of the external and internal popliteal nerves for paralysis of all muscles supplied by the former so long ago as 1905.

My friend Spitzy of Graz has published a most interesting series of papers, rumning from 1904 to the present day, in which he has dealt with the subject carefully, both from the experimental and clinical point of view. Nevertheless, I come before you with very slight personal experience of the operation, not sufficient to speak with any authority.

I would put forward with all diffidence a general criticism of reported cases. Many surgeons have performed the operation in a few cases, and have reported a few successes and many failures. Of the failures I shall speak later, but shali first say a word of the successes.

So far as. I have seen reports of successful cases, the procedure has been to perform the operation of nervo tiransplantation, and then fix the limb in a position which woild keep the grafted nerve relaxed. At the same time the muscles have been assiduously massaged, and every means taken to get them into good condition. In this lurks the fallacy that the success, in some instances at any rate, may be due to the muscles having been in a state of functional disability, and not really paralysed at all.

The author's of these reports do not prove to my satisfaction that every means has previously been taken to exclude this possibility. I must remind you of Wittek's case, whicl I have quoted, and of my own many experiences of instances where recovery of power has taken place unexpectedly, but. in absolute accord with the principles we have:discussed.

I by no means intend this criticism to mean that none of these successes are credible, but that they are lacking in scientific value, because sufficient care has not been taken to exclude the fallacy which I have indicated.

I may be charged with lack of enterprise for not having performed the operation, on a series of cases in which 1 have been contented with arthrodesis, after satisfying myself that no recovery is likely to occur in the paralysed group. My answer to this charge is a very simple oneI do not know how to perform the operation with any degrce of accuracy or certainty. Consider a case of paralysis of the muscles supplied by the anterior tibial nerre. A successful neuroplasty demands that the bundle of motor nerves intended for this group of muscles bc isolated from the tiunk of the external popliteal nerve and grafted into an active motor bundlc in the internal popliteal. I speak of bundles in this way, for six ycars ago Spitzy pointed ont that in operating on the anterior crural nerve where it enters the thigh it must be rememberect that all the motor fibres lie in the outer half of the nerve, and all the sensory fibres in the inner half. Stoeffel has recently published a most valuable anatomical research on this subject, in which he has shown that both in the upper and lower limb nerres to groups of muscles-for example, the anterior tibial nerve-can be traced back well up the main trunk as a distinct bundle wrapped in their own sheath. He has shown, too, that in the case of the musculocutaneous nerve in the leg, the motor fibres to the perone1, and the sensory branches can be treated as separate bundles, not only in the trunk of the musculo-cutaneous nerve, but also up into the truuk of the external popliteal nerve.

In the face of sueh anatomical difficulties it is not surprising that the results of nerve transplantation for the treatment of paralysis have been uncertain and disappointing. If Stoeffel, and others working on the same lines, can give us a clear account of the internal topography of nerve trunks, and show us how we can identify with certainty bundles of nerres destined to supply particular groups of muscles, the first step will lrave becn taken towards putting neuroplasty on a sound scientific footing.

The subject of the treatment of polionyelitis is so farreaching that, in spite of this long address, I have only becn able to touch lightly and inadequately upon a few of its many points of interest. It offer's a most fascinating field, full of variety to a surgeon who has patience and enthusiasm. To approach the subject in a spirit of (loubt and pessimism is fatal to success.

\section{A Host-(braduate lerture}

\section{NEUROTIC DYSPEPSIA.}

Defrerey at the London School of

Clinical Medicine.

BY GL'THRIE RANKIN, M.D., F.R.C.P.,

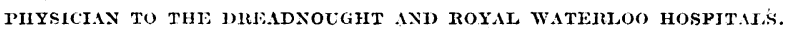

You may remember that a week or two ago wo investigated, a case of gastric disturbance in a man, about whom wo came to the concinsion that he was probably suffering from a local functional condition grafted on to a general state of neurasthenia, sinulating at first sight, in many of the subjective symptoms and the objective signs, an ulcer either of the duodenum or of the pyloric end of the stomach. Though cases of this description are not often seen in this hospital, they are common in private practice, and the more I see of them the more I become convinced! of the necd for caution in arriving at a firm diagnosi; until full opportunity has been afforded of watching the progress of events under the influence of rest, dietary, anc' drugs. This occasion is opportune for bringing to you notico some recent cases coming within my own experience which testify to the need for such an attitude of expectant observation. The classification of functional disorders of the digestive organs is so confused that the difficulties of diagnosis are often very considerable. But, when we come to think of it, the fumotion of any organ can be disturbeci only in one of three ways-by excess, by default, or. by perversion-and, in accordance with this propesition, Professor Robin's suggestion that all dyspepsias - should be grouped under three primary headings, according as they are caused by hyperfunction, hypofunction, or abnormal fermentations, has much to recommend it. While the great bulk of dyspepsias may, in a broad way, be parcelled out into these three varieties. it is obvious that there must be much overlapping of one class of case with another; that a patient may present such a succession of symptoms as would bring him into one category of the disease at one time and into another at another; and that there must be certain secondary groups of the disorder so well marked as 